\title{
Eunethydis 1st international ADHD conference: from data to best clinical practice
}

\author{
26th-28th May 2010, Amsterdam, The Netherlands
}

(c) Springer-Verlag 2010

\section{ORGANISATION/COMMITTEES}

\section{Organisation}

European Network for Hyperkinetic Disorders (Eunethydis)

Scientific Programme Committee

Prof. Joseph Sergeant, Eunethydis and Scientific

Committee Chairman, The Netherlands

Prof. Tobias Banaschewski, Germany

Dr. David Coghill, UK

Prof. Edmund Sonuga-Barke, UK/Belgium

Prof. Eric Taylor, UK

Prof. Alessandro Zuddas, Italy

\section{Guest editor:}

Prof. Joseph A. Sergeant

VU University Amsterdam

Faculty of Psychology and Education

Van der Boechorststraat 1

1081 BT Amsterdam

The Netherlands

E-mail: JA.Sergeant@psy.vu.nl

\section{CONTENTS}

Editorial

Main Conference Lectures

Early Career Lectures

Poster Sessions
A. Diagnosis, Evaluation and Treatment
B. Cognitive-Energetic Factors in ADHD
C. Endophenotypes and Genetic Factors
D. Reward Processing
E. Neuropsychological Issues
F. Pharmacological and Non-pharmacological Treatment Issues
G. Emotional Factors
H. Miscellaneous/Late Submissions
Junior Conference Lectures 